\title{
Ionic Motion in Polypyrrole-Cellulose Composites: Trap Release Mechanism during Potentiostatic Reduction
}

\author{
Maria Strømme, ${ }^{*}, \dagger$ Göran Frenning, ${ }^{\ddagger}$ Aamir Razaq, ${ }^{\dagger}$ Kristina Gelin, ${ }^{\S}$ Leif Nyholm,, and \\ Albert Mihranyan ${ }^{\dagger}$ \\ Nanotechnology and Functional Materials, Department of Engineering Sciences, The Ångström Laboratory, \\ Uppsala University, Box 534, 75121 Uppsala, Sweden, Department of Pharmacy, Uppsala Biomedical Centre, \\ Uppsala University, Box 580, 75123 Uppsala, Sweden, Department of Physical and Analytical Chemistry, \\ Uppsala University, P.O. Box 579, SE-751 23 Uppsala, Sweden, and Department of Materials Chemistry, The \\ Angström Laboratory, Uppsala University, Box 538, 75121 Uppsala, Sweden
}

Received: January 11, 2009; Revised Manuscript Received: February 09, 2009

\begin{abstract}
This work investigates the movement of anions during potentiostatic controlled reduction of novel composite materials consisting of high surface area cellulose substrates, extracted from the Cladophora sp. algae, coated with thin $(\sim 50 \mathrm{~nm})$ layers of the intrinsically conducting polymer (ICP) polypyrrole. The coating was achieved by chemical polymerization of pyrrole on the cellulose fibers with iron(III) chloride and phosphomolybdic acid, respectively. The composites are in the form of paper sheets and can be directly immersed into an electrolyte solution for ion absorption/desorption. The motion of glutamate and aspartate anions during cathodic polarization was investigated as a function of preceding anodic polarization at various potentials. The composite was found to exhibit memory effect as the response to a cathodic polarization of constant magnitude produced different responses depending on the magnitude of the preceding anodic potential. After the application of a cathodic potential to the composite, the reduction current curves-generated by anions leaving the composite-were found to initially increase in magnitude followed by a monotonic decay. A similar response has not been described and analyzed for electrochemical reduction of anion containing ICP materials earlier. A theoretical model was developed to aid the analysis of the experimental data. The model accounts for both freely mobile anions and anions that may be temporarily trapped in a contracting PPy network during cathodic polarization. By fitting the recorded reduction current curves to this model, detailed information about the ionic movement in the composite could be obtained, which may be used to further optimize the materials properties of conducting polymer systems aimed for specific electrochemical ion exchange processes.
\end{abstract}

\section{Introduction}

The conductive properties of polyacetylene resulted in the 2000 Nobel Prize in Chemistry, and various analogues of it have been investigated throughout the years including polyphenylene, polyphenylene sulfide, polyphenylene vinylene, polypyrrole, polythiophene, and polyaniline. ${ }^{1-3}$ Polypyrrole (PPy) and polyaniline are probably the most promising of currently known conductive polymers in a number of applications due to their reasonably high conductivity, good stability of the oxidized state, and ease of processing. Of the two, PPy is often preferred due to its superior electrical conductivity and facile synthesis both in aqueous and organic media.

During the chemical polymerization of pyrrole, anions in the electrolyte solution are incorporated in the polymer film to maintain charge balance. The presence of these so-called dopant ions greatly influences the properties of the film. It is generally conceived that both anions and cations as well as accompanying water can enter the polymer film upon oxidation and reduction. ${ }^{4-7}$ If a small anion with high mobility is incorporated into a polymer film as a dopant during polymerization, it can be expelled when the polymer is reduced. The ion-exchange

\footnotetext{
* Corresponding author:maria.stromme@angstrom.uu.se.

${ }^{\dagger}$ Department of Engineering Sciences, Uppsala University.

* Department of Pharmacy, Uppsala University.

$\S$ Department of Physical and Analytical Chemistry, Uppsala University.

" Department of Materials Chemistry, Uppsala University.
}

properties are largely dependent on the character of the ion being exchanged, e.g., on size, charge, state of hydration. Usually large organic anions or relatively small but double-charged inorganic anions exhibit lower mobility. ${ }^{8}$ By doping the film with large anions, which have mobilities low enough to confine them inside the polymer, one can reversibly absorb and desorb cations which will be attracted to the large anions to maintain charge neutrality. Various biologically active entities such as enzymes, ${ }^{9,10}$ antibodies for immunosensors, ${ }^{11}$ or metal complexing entities ${ }^{12}$ can be incorporated in PPy films for highly specific molecular and/or ionic recognition and separation. This phenomenon lays the foundation for various applications of PPy films in ion exchange membranes and separation, ${ }^{13-16}$ wherein the adsorption and desorption of valuable entities is achieved merely by varying an electrochemical potential.

The fundamental principles governing the ion transport in the conducting polymer films as a function of applied potential are complex. It is conceived that when an anodic (positive) potential is applied to a completely reduced film, a propagating boundary between the insulating (discharged) and conducting (charged) phases is built. This process is associated by a propagation of conducting paths in the polymer film resembling percolation effects. The movement of the propagating conductive zone from the tip of the electrode to the edges was experimentally verified in a series of works by Aoki and coauthors. ${ }^{17-19}$ The build-up of the conducting paths in the film is paralleled by an inflow of oppositely charged anions into the film aimed 


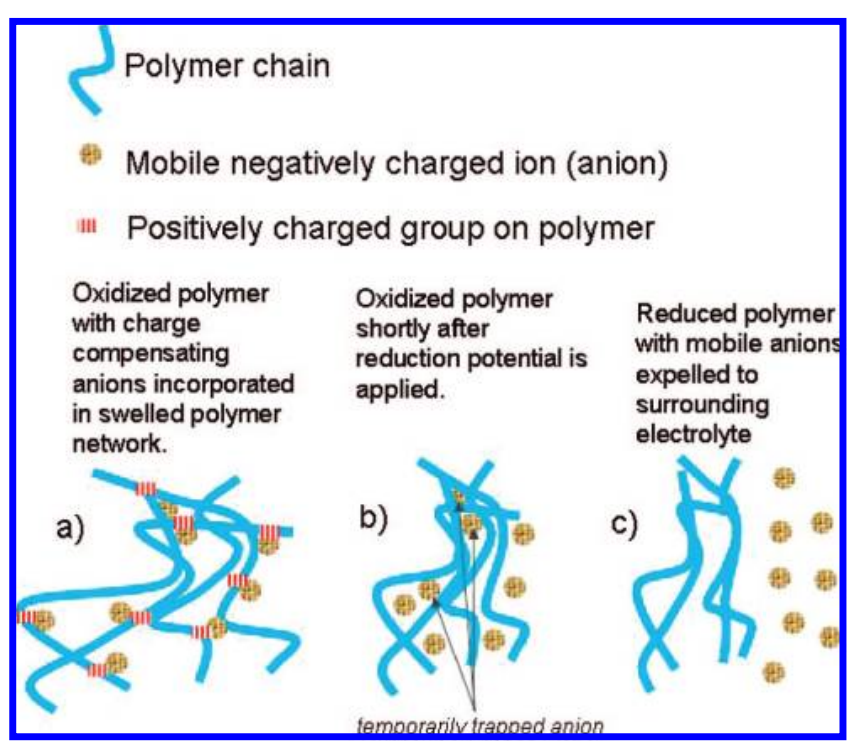

Figure 1. Schematic illustration showing the response of mobile anions and polymer network to applied potential. (a) The polymer is in the oxidized state. The groups on the polymer chain are positively charged, and anions are incorporated from the surrounding electrolyte to charge balance these groups. Upon anion incorporation the polymer network swells. (b) A reducing potential is applied to the oxidized polymer. Some anions are immediately released and move to the surrounding electrolyte when the positively charged groups are reduced. As result of this reduction step the polymer network contracts and some anions become temporarily trapped in the contracting polymer network. (c) An equilibrium state in the reduction process is reached. The polymer network is contracted, and all mobile anions have left the polymer for the surrounding electrolyte.

at compensating the charge imbalance. Therefore, whereas at the initial stages of an anodic step the current may increase in its magnitude due to the overall increase in the number of conducting paths, the latter is invariably followed by decrease in the current due to a diffusion of charged species in the film. If, however, a cathodic potential is applied to the film in an oxidized state, the film is rendered insulating properties. Unlike the anodic process, wherein there is a clear propagation of the boundary between the conductive (charged) and insulating (discharged) zones from the tip of the electrode to the edges, no such boundary is observed upon reduction, and residual charge islands exist in the form of spatially restricted clusters. ${ }^{20}$ The conduction paths between these clusters and the electrode are broken so that the material can only be discharged during a long-time film exposure at a cathodic potential or by chemical reduction. The ability of the film to reversibly absorb and desorb charged species over an extended number of redox cycles would determine the usefulness of the film as an ion-exchange medium.

During anodic polarization, electrostatic repulsions occur between the neighboring positively charged polymer chains. ${ }^{21}$ As a result, anions from the electrolyte solution are driven into the film to maintain electroneutrality causing the polymer network to swell. Upon application of the cathodic potential, the ions are being expelled from the polymer and the polymer network contracts. The latter phenomenon lays the foundation for use of conductive polymers in artificial muscle applications. ${ }^{22}$ It can thus be concluded that the cathodic potential controls the degree of compactness of the molecular entanglement of conductive polymer film, whereas the anodic potential, once the chain relaxation commenced, controls the extent of oxidation. ${ }^{23}$ Figure 1 illustrates the response of the mobile anions and the polymer network to an applied potential. In this figure only the mobile anions as well as the positively charged polymer groups belonging to conducting paths that span through the entire polymer are depicted. Isolated conducting clusters and anions completely trapped in the network are thus not considered.

The thickness of the conductive polymer is also of importance for efficient ion-exchange as the ions, particularly those with restricted mobility, are capable of penetrating only short distances into the polymer matrix. Thin coating distributed over a large internal surface area is more efficient for ion exchange capacity than thick coating on an essentially nonporous substrate. We have recently described a novel composite material consisting of high surface area cellulose, extracted from the Cladophora sp. algae, and thin $(\sim 50 \mathrm{~nm})$ PPy coating, featured with high ion-exchange capacity. ${ }^{24-26}$ The material is in the form of paper sheets, which can be directly immersed into the electrolyte solution for ion exchange. It was shown that the size (or molecular weight) of the oxidizing agent used for polymerization of pyrrole affected both the ion incorporation capacity and the size selectivity of the ion incorporation.

In this work we present a theoretical model describing the flux of anions from a conducting polymer layer upon contraction of the polymer network during potentiostatic controlled reduction of the polymer. The model contains a parameter describing the relative portion of anions that are temporarily trapped in the contracting polymer network (Figure 1b) and anions that are free to diffuse out to the surrounding electrolyte right after application of the reduction potential. It also contains a parameter describing the frequency by which the temporarily trapped anions are released as well as the anion diffusion coefficient. The model is employed to fit experimental data obtained from reduction of PPy coated Cladophora cellulose samples.

\section{Materials and Methods}

Chemicals and Reagents. Cladophora algae were collected from the Baltic Sea. The cellulose was extracted from Cladophora algae as described previously. ${ }^{27}$ Pyrrole (Py), iron(III) chloride $\left(\mathrm{FeCl}_{3}\right)$, phosphomolybdic acid hydrate (PMo), and hydrochloric acid $(\mathrm{HCl})$ were used as supplied by VWR, Sweden. DL-Aspartic acid (99\%) and DL-glutamic acid (98\%) were purchased from Sigma Aldrich USA. Fresh Py was used for synthesis.

Preparation of the Composite Material. Three different compositions were prepared for the present study: PPy-coated samples synthesized by using iron(III) chloride as the oxidizing agent containing either (i) glutamate $\left(\mathrm{PPy} / \mathrm{FeCl}_{3}\right.$-glut) or (ii) aspartate (PPy/PMo-asp) as the mobile anions and (iii) $\mathrm{PPy}$ coated samples synthesized by using PMo as the oxidizing agent and containing glutamate as the mobile anion (PPy/PMo-glut).

The samples were prepared as described in Razaq et al. ${ }^{25}$ Briefly, $300 \mathrm{mg}$ of Cladophora cellulose powder was dispersed in $50 \mathrm{~mL}$ of water using high energy-ultrasonic treatment (VibraCell 750W, Sonics, USA) for $8 \mathrm{~min}$, and the dispersion was collected on a filter paper. Py $(3 \mathrm{~mL})$ was put in a volumetric flask, and the total volume was brought to $100 \mathrm{~mL}$. The collected cellulose cake was mixed with Py solution and dispersed using ultrasonicator for $1 \mathrm{~min}$. The dispersion was allowed to stand for $30 \mathrm{~min}$ and then collected on filter paper.

To prepare the iron(III) chloride oxidized samples $8 \mathrm{~g}$ of $\mathrm{FeCl}_{3}$ was dissolved in $100 \mathrm{~g}$ of water and run through the filter cake to induce polymerization (the reaction was allowed to continue for $10 \mathrm{~min}$ prior to filtration). A fluffy sponge like cake was formed. $100 \mathrm{~mL}$ of $0.1 \mathrm{M} \mathrm{HCl}$ was run through the cake. The product was then thoroughly washed with water and dried (the cake was redispersed using ultrasonicator to form a 
homogeneous layer). The same procedure was followed to make the PMo-oxidized samples, but for these, PMo $(34 \mathrm{~g} / 100 \mathrm{~mL})$ replaced the $\mathrm{FeCl}_{3}$ and no $\mathrm{HCl}$ was used.

Conductivity Measurements. The conductivity of the samples was measured at room temperature using a semiconductor device analyzer (B1500A, Agilent Technologies, USA). Prior to the measurements, silver paint was pasted at the ends of rectangular samples to ensure good contacts.

Electrochemical Sample Preparation and Analysis. The two glutamate containing compositions, $\mathrm{PPy} / \mathrm{FeCl}_{3}$-glut and $\mathrm{PPy} /$ PMo-glut, under study were electrochemically prepared in an electrolyte solution containing $2 \mathrm{M}$ of DL-glutamic acid ( $\mathrm{pH}$ 9.0). The PPy-functionalized Cladophora cellulose samples (either oxidized with $\mathrm{FeCl}_{3}$ or PMo) were first reduced for $300 \mathrm{~s}$ at a cathodic potential of $-0.8 \mathrm{~V}$, then oxidized for $300 \mathrm{~s}$ at anodic potential of $0.7 \mathrm{~V}$, and then reduced again for $600 \mathrm{~s}$ at the cathodic potential of $-0.8 \mathrm{~V}$. After this, different anodic potential steps were applied by increasing the oxidation potential from 0.7 to $1.2 \mathrm{~V}$ for $300 \mathrm{~s}$, thus preparing samples containing an increasing amount of glutamate anions. ${ }^{25}$ The samples were reduced between each anodic potential step at $-0.8 \mathrm{~V}$ for $300 \mathrm{~s}$, and the current measured during these reduction steps generated the experimental data used in the analysis of the present work

The aspartate compositions, $\mathrm{PPy} / \mathrm{FeCl}_{3}$-asp, under study were electrochemically prepared by treating PPy functionalized Cladophora cellulose samples oxidized with $\mathrm{FeCl}_{3}$ in the same manner as described above by replacing the $2 \mathrm{M}$ DL-glutamic acid electrolyte with a DL-aspartic acid ( $\mathrm{pH}$ 10.4) of similar concentration.

The electrochemical experiments were carried out using a standard 3 electrode electrochemical cell employing an Autolab/ GPES interface (ECO Chemie, The Netherlands) with the sample as the working electrode, a $\mathrm{Pt}$ wire as the counter electrode, and an $\mathrm{Ag} / \mathrm{AgCl}$ electrode as the reference. All measurements were carried out at room temperature. The typical dimensions of the samples used in the electrochemical measurements were $0.6 \mathrm{~cm} \times 0.25 \mathrm{~cm} \times(0.06-0.2 \mathrm{~cm})$, which corresponded to sample weights of $\sim 10 \mathrm{mg}$.

Scanning Electron Microscopy (SEM). Micrographs were taken with an environmental SEM (FEI/Philips XL 30, The Netherlands) in the high vacuum mode. The samples were mounted on aluminum stubs using double-sided adhesive tape. Prior to imaging $\mathrm{Au} / \mathrm{Pt}$ was sputtered on the samples to minimize charging.

\section{Theory}

To derive an analytical expression for the reduction current, our starting point is the one-dimensional inhomogeneous diffusion equation

$$
\frac{\partial C}{\partial t}=D \frac{\partial^{2} C}{\partial x^{2}}+\rho
$$

where $D$ is the diffusion coefficient of the mobile anions and the mobile anion concentration $C(x, t)$ is assumed to be a function of the spatial coordinate $x$ and time $t$ after the reduction potential was applied. To account for the fact that a portion of the mobile anions may be temporarily trapped in the contracting polymer network when the reduction potential is applied, a source term $\rho(t)$ describing the release of these ions is introduced. This source term is assumed to be a function of time only, of the form

$$
\rho=v R e^{-v t}
$$

where $v$ is a release frequency and $R$ is the total amount of anions being released per volume unit (this assertion may be verified by integration of eq 2 over all times $t>0$ ). The material is assumed to occupy the region in space between $x=0$ and $x$ $=L$, with a no-flux boundary condition at the former boundary and sink conditions at the latter; hence the appropriate boundary conditions are

$$
\begin{gathered}
\frac{\partial C}{\partial x}=0 \text { at } x=0 \\
C=0 \text { at } x=L
\end{gathered}
$$

With the above arrangement the composite under study, that may release anions from both sides of the sample, should be regarded to have a thickness $L$ equal to half its macroscopic thickness and the area $A$ of the composite should be regarded as the double of the actual macrocopic sample area.

In the derivation that follows, we assume that a portion of the mobile anions are untrapped and immediately free to move when the reduction potential is applied. We further assume that these immediately mobile anions have a uniform initial concentration (i.e., that $C=C_{0}$ for $t=0$ ) but will subsequently generalize the obtained result somewhat (see below).

The solution to the described problem is most conveniently obtained by using Laplace transformation. Taking the initial condition into account, eq 1 is transformed into

$$
D \frac{d^{2} \tilde{C}}{d x^{2}}-s \tilde{C}=-\left(C_{0}+\frac{v R}{s+v}\right)
$$

where $\tilde{C}(x, s)$ is the Laplace transform of $C(x, t)$ and where $s$ denotes the Laplace transform variable. Equation 5 is an ordinary differential equation for $\tilde{C}$ that may readily be solved by standard methods; the solution that fulfils the boundary conditions 3 and 4 may be expressed as

$$
\tilde{C}=\frac{1}{s}\left(C_{0}+\frac{v R}{s+v}\right)\left[1-\frac{\cosh (x \sqrt{s / D})}{\cosh (L \sqrt{s / D})}\right]
$$

The Laplace transformed flux out of the sample (i.e., $-D d \tilde{C} /$ $d x$ evaluated at $x=L$ ) thus becomes

$$
\tilde{J}(S)=\frac{1}{\sqrt{s / D}}\left(C_{0}+\frac{v R}{s+v}\right) \tanh (L \sqrt{s / D})
$$

whereas the flux $J(t)$ itself is obtained via the inverse Laplace transform

$$
J(t)=\frac{1}{2 \pi i} \int_{\gamma-\mathrm{i} \infty}^{\gamma+\mathrm{i} \infty} \tilde{J}(s) e^{s t} d s
$$

where $\gamma$ is chosen so that all singularities of the integrand are to the left of the contour. The Bromwich integral (eq 8) may be readily evaluated by using residue calculus. Let us to this end define the two functions 


$$
p(s)=\frac{\left[(s+v) C_{0}+v R\right] \sinh (L \sqrt{s / D}) e^{s t}}{\sqrt{s / D}}
$$

and

$$
q(s)=(s+v) \cosh (L \sqrt{s / D})
$$

so that the integrand $\tilde{J}(s) e^{s t}=p(s) / q(s)$. The function $p(s)$ is analytic everywhere in the complex plane (note that $\sinh (L \sqrt{ } s /$ $D) \cong L \sqrt{ } s / D$ in a neighborhood of the origin). The function $q(s)$ clearly has a zero at $s_{0}=-v$, and the cosh factor produces an infinite number of zeroes located at

$$
s_{n}=-\left(n-\frac{1}{2}\right)^{2} \frac{\pi^{2} D}{L^{2}} \quad n=1,2,3 \ldots
$$

In what follows, we will assume that all zeroes are distinct, which is the case except for some special values of $v$. The corresponding residues may then be evaluated as $p\left(s_{n}\right) / q^{\prime}\left(s_{n}\right)$ (where the prime denotes differentiation). For convenience defining $\alpha=L \sqrt{ } v / D$ and letting $\mu_{n}=-s_{n}(n=1,2,3, \ldots)$ to clearly highlight the fact that the exponents are negative, we thus obtain

$$
\operatorname{Res}_{s=s_{n}}\left[\tilde{J}(s) e^{s t}\right]=\left\{\begin{array}{cc}
\frac{D R}{L} \alpha \tan (\alpha) e^{-v t} & n=0 \\
\frac{2 D}{L}\left(C_{0}-\frac{R}{\mu_{n} / v-1}\right) e^{-\mu_{n} t} & n=1,2,3, \ldots
\end{array}\right.
$$

By use of a standard procedure involving the residue theorem, ${ }^{28}$ we find that the flux equals the sum of the above residues, i.e.,

$$
J(t)=\sum_{n=0}^{\infty} \operatorname{Res}_{s=s_{n}}\left[\tilde{J}(s) e^{s t}\right]
$$

Equations 12 and 13 between them constitute the desired solution.

Since the system response is linear, it is evident that the flux may be decomposed as $J=J_{0}+J_{1}$, where

$$
J_{0}(t)=2 \frac{D C_{0}}{L} \sum_{n=1}^{\infty} e^{-\mu_{n} t}
$$

and

$$
J_{1}(t)=\frac{D R}{L}\left[\alpha \tan (\alpha) e^{-v t}-2 \sum_{n=1}^{\infty} \frac{e^{-\mu_{n} t}}{\mu_{n} / v-1}\right]
$$

are the fluxes resulting from the immediately mobile anions and the anions being released, respectively. This decomposition enables us to generalize the initial condition somewhat: A uniform initial concentration of immediately mobile anions (as assumed up to this point) would correspond to an infinite concentration gradient at $x=L$ at $t=0$, which would result in an infinite initial flux. To ensure that the flux always is finite, we introduce a lag time $\tau$ and replace eq 14 with

$$
\hat{J}_{0}(t)=2 \frac{D C_{0}}{L} \sum_{n=1}^{\infty} e^{-\mu_{n}(t+\tau)}
$$

Physically, eq 16 corresponds to an initial immediately mobile anion concentration that equals the concentration profile that would have been obtained at time $t=\tau$ for purely diffusional release (i.e., no trapped ions) from a sample containing a uniform amount of mobile anions at $t=0$. Since the lag time thus introduces a concentration gradient at the sample boundary, the initial amount of mobile ions present in the sample will be a factor $\varphi$ smaller than for a uniform initial ion concentration. By use of eqs 14 and 16, it may be found that

$$
\varphi=2 \sum_{n=1}^{\infty} \frac{e^{-(n-1 / 2)^{2} \pi^{2} \beta}}{(n-1 / 2)^{2} \pi^{2}}
$$

where we have let $\beta=D \tau / L^{2}$.

The measured reduction current $I$ of a composite of macroscopic area $A / 2$ and thickness $2 L$ could thus be fitted to

$$
\begin{array}{r}
I=q A\left\{\frac{D R}{L}\left[\alpha \tan (\alpha) e^{-v t}-2 \sum_{n=1}^{\infty} \frac{e^{-\mu_{n} t}}{\mu_{n} / v-1}\right]+\right. \\
\left.2 \frac{D C_{0}}{L} \sum_{n=1}^{\infty} e^{-\mu_{n}(t+\tau)}\right\}
\end{array}
$$

according to the above presented model.

From eq 18 the parameter $m$, describing the relative portion of mobile anions that are immediately free to move when the reduction potential is applied, can be obtained as

$$
m=\frac{\varphi C_{0}}{\varphi C_{0}+R}
$$

\section{Results and Discussion}

Figure 2 shows SEM images of the iron(III) chloride and PMo-synthesized composites. The iron(III) chloride synthesized sample (panel a) displays the fine fibril structure which is typical for Cladophora cellulose. ${ }^{29}$ For the PMo-synthesized composites, this fibril structure does not seem to be fully preserved as the samples exhibit a more nodular cauliflower-like morphology (panel b). As shown previously, ${ }^{25}$ transmission electron microscopy analysis of the samples showed that the PPy layer covering the cellulose fibrils was approximately $50 \mathrm{~nm}$ thick for both samples thus creating fibers with a diameter slightly larger than $100 \mathrm{~nm}$.

The conductivity of the $\mathrm{PPy} / \mathrm{FeCl}_{3}$ samples was $0.65 \mathrm{~S} / \mathrm{cm}$, whereas the corresponding value for the PPy/PMo samples was $0.12 \mathrm{~S} / \mathrm{cm}$.

Parts a $-\mathrm{c}$ of Figure 3 show the current curves recorded during cathodic polarization at $-0.8 \mathrm{~V}$ of the samples under study after the samples had been anodically polarized at the displayed potentials. All reduction current curves for the glutamatecontaining samples as well as the reduction current curves for the aspartate containing sample following oxidation at the highest potentials show an initial increase in magnitude followed by a monotonic decay.

An initial current increase followed by a decay is often seen during constant potential anodic polarization of PPy films ${ }^{23-26,30,31}$ 


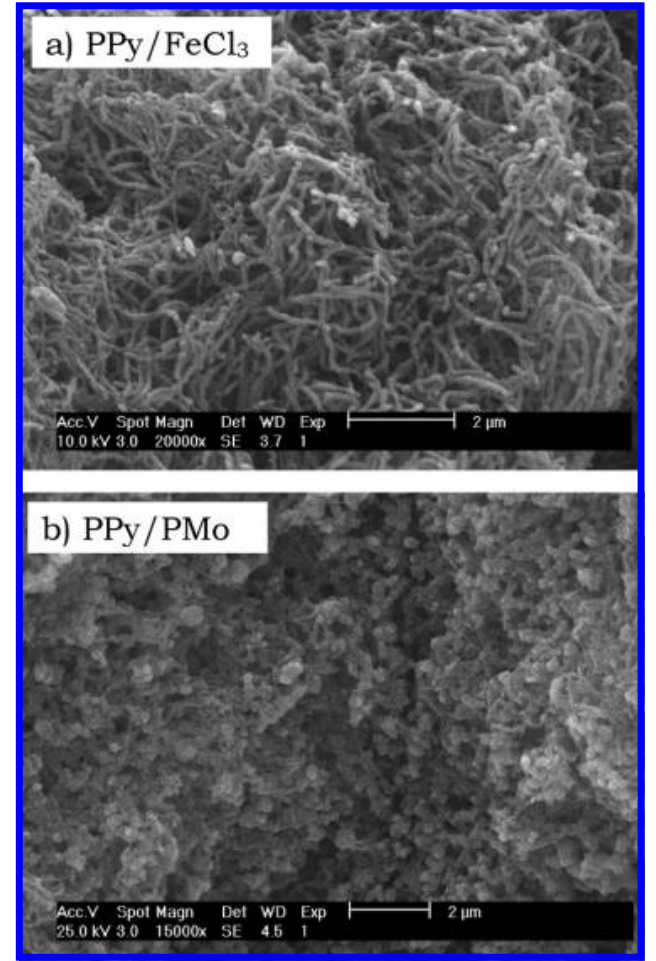

Figure 2. SEM micrographs of polypyrrole/Cladophora cellulose composites synthesized with (a) iron(III) chloride and (b) PMo.

and can be ascribed to the nucleation of conducting paths in the polymer followed by diffusion of anions into the polymer. To our knowledge, a similar response has not been presented and analyzed for the reduction of anion containing samples. During reduction of the samples the anions incorporated during oxidation are expelled and the polymer network contracts. Therefore, the initial increase in magnitude of the current may be ascribed to a release process in which some of the mobile anions are trapped in the contracting polymer network and released in a time dependent manner, eq 2. Similar trap release processes have been used to describe the ionic current response to a constant potential at the electrolyte/oxide interface in electrochemical setups ${ }^{32}$ as well as from interfacial traps between an electrode and a dielectric material. ${ }^{33}$

By fitting the analytical expression, eq 18, derived for the cathodic current response of a system consisting of both initially trapped and initially mobile anions, to the experimental current responses in parts $\mathrm{a}-\mathrm{c}$ of Figure 3 , detailed information about the ionic movement in the composite could be obtained. As exemplified in parts $\mathrm{d}$ and e of Figure 3, eq 18 described the measured current response very well, and the extracted fitting parameters $D, m$, and $v$ are displayed in Figure 4 . To simplify the interpretation of these data, the number of ions incorporated in the composites under study during the anodic polarization preceding the cathodic process is displayed in Figure 5. These data were presented and discussed in detail previously. ${ }^{25}$ From the latter figure we mainly observe that an anodic potential of $+0.7 \mathrm{~V}$ was too low to fully oxidize the $\mathrm{PPy} / \mathrm{FeCl}_{3}$ sample using glutamate ions and that otherwise the order of magnitude of incorporated ions was relatively insensitive to the oxidation potential used except from a slight evidence of overoxidation and thus a decrease in number of incorporated ions at the highest potential in the $\mathrm{PPy} / \mathrm{FeCl}_{3}$ composite containing aspartate ions.

Above and in the following, it is assumed that the reduction current is controlled by the rate of the anion desorption process. This is a reasonable assumption since it has previously been found $^{34}$ that no charge compensation by absorption of cations could be detected in electrolytes containing anions with a diameter smaller than or equal to that of $\mathrm{PO}_{4}{ }^{3-}$ (viz. $\sim 5 \AA^{35}$ ) during the reduction of the oxidized polymer with PPy covered electrodes synthesized in the presence of $\mathrm{ClO}_{4}{ }^{-}$ions. Since glutamate and aspartate anions are of similar size as $\mathrm{PO}_{4}{ }^{3-}$ and given the thin PPy coatings used, it is reasonable to assume that cation absorption can be totally neglected during the reduction of the presently studied materials. This assumption is further supported by previous findings, ${ }^{25}$ indicating that the anions under study occupy sites in the close vicinity of the PPy surface and should therefore be able to readily leave the sample during the reduction step. The influence of cation absorption on the behavior of the PPy samples will therefore not be further considered here.

From Figure 4a we observe that the diffusion coefficients for both the glutamate and the aspartate ions in the $\mathrm{PPy} / \mathrm{FeCl}_{3}$ composite were of the order of $10^{-5} \mathrm{~cm}^{2} / \mathrm{s}$, which is similar to ionic diffusion coefficients in free water, whereas the diffusion coefficient for the glutamate ions in the PPy/PMo composite was between 1 and 2 orders of magnitude lower. The presented diffusion coefficients are effective values representing the speed of diffusion of the anions from the position they were situated in at the end of the oxidation process and until they have reached the bulk electrolyte during the reduction process. The fact that the diffusion coefficient for the ions in the $\mathrm{PPy} / \mathrm{FeCl}_{3}$ composite is similar to that in free water supports the above-discussed results presented earlier ${ }^{25}$ indicating that only polymer sites very close to the polymer/electrolyte interface were accessed by the ions during oxidation. Therefore, the main contribution to the effective diffusion coefficient comes from movement of the ions in electrolyte filled pores in the composite as opposed to movement inside the polymer layer. The PPy/PMo composite, which was produced using phosphomolybdate anions as oxidizing agent, is expected to contain vacancies of the order of 10 $\AA^{36}$ before the glutamate ions are inserted into the composite during oxidation. These vacancies are thus expectedly much larger than those produced by the chloride anions used as oxidizing agent in the production of the $\mathrm{PPy} / \mathrm{FeCl}_{3}$ sample. It is therefore likely that the glutamate ions more easily can access sites further away from the polymer/electrolyte interface and into the polymer in the PPy/PMo composite than in the PPy/ $\mathrm{FeCl}_{3}$ composite. This is expected to lead to a larger contribution from diffusion inside the polymer and thus a lower effective diffusion coefficient upon anion extraction during reduction. The fact that the glutamate diffusion coefficient decreases after the $\mathrm{PPy} / \mathrm{FeCl}_{3}$ composite has been oxidized at the highest oxidation potentials used in this study $(+1.2$ and $+1.3 \mathrm{~V})$ may indicate that a portion of the incorporated anions was able to move further into the polymer during oxidation at these potentials than at the lower ones. Thus, the contribution from diffusion inside the polymer becomes larger during ion extraction after oxidation at these higher potentials.

Figure $4 \mathrm{~b}$ shows that about $20 \%$ of the ions extracted during reduction are free to move immediately after the reduction potential is applied to both the glutamate and the aspartate containing $\mathrm{PPy} / \mathrm{FeCl}_{3}$ composite, when the oxidation potential used to insert the ions was above $+0.8 \mathrm{~V}$. For the glutamate ions inside the PPy/PMo composite the situation is different; when oxidation potentials below $\sim+1 \mathrm{~V}$ were applied, about $20 \%$ of the ions were free to move immediately after the reduction potential was applied. But after oxidation at higher potentials, an increasing portion of immediately free ions is observed. At these higher potential the magnitude of the 


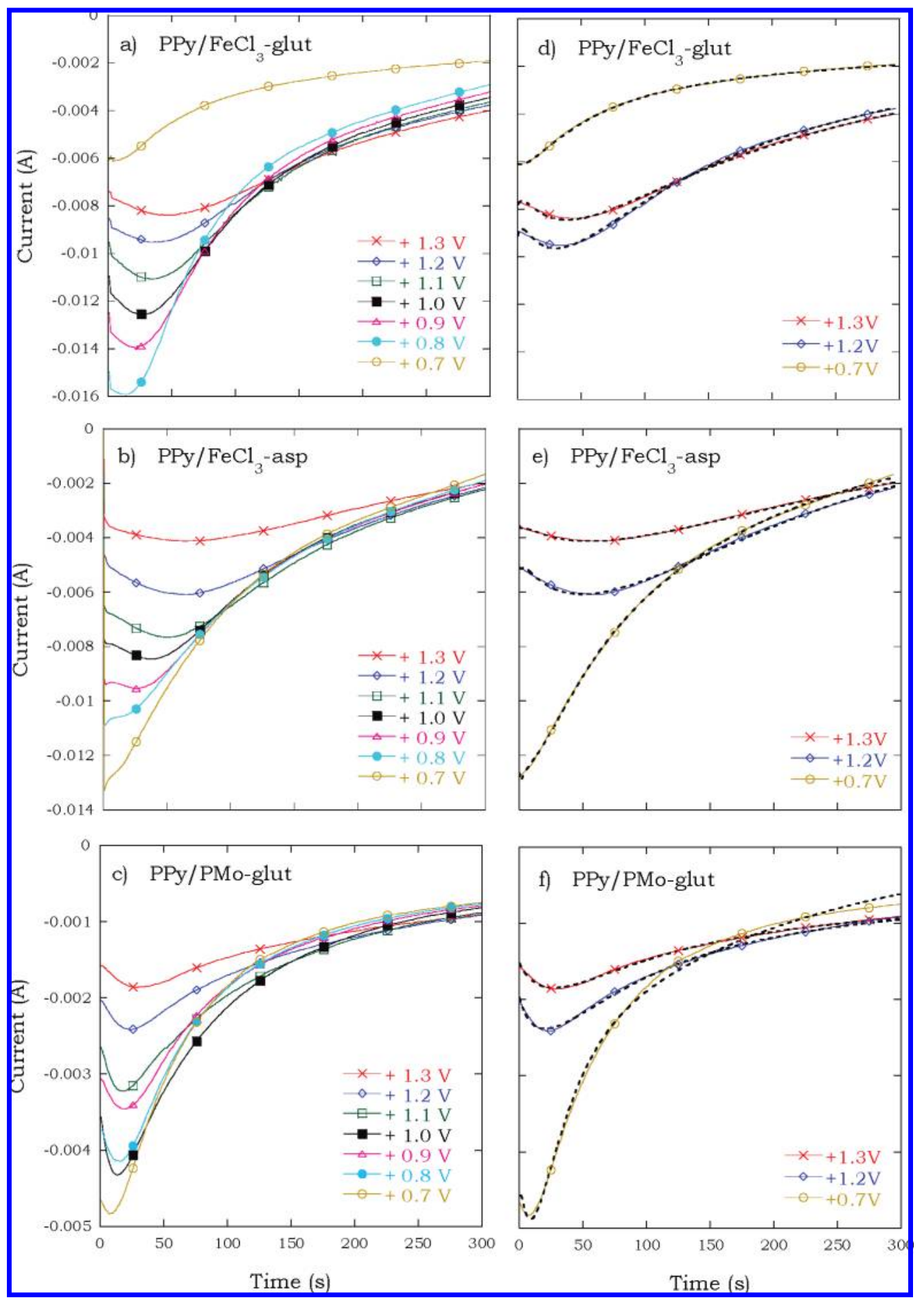

Figure 3. Measured current during cathodic polarization $(-0.8 \mathrm{~V}$ vs $\mathrm{Ag} / \mathrm{AgCl})$ after oxidation at the displayed potentials for $300 \mathrm{~s}$ in electrolytes containing $2 \mathrm{M}$ of glutamate ions (a and c) or aspartate ions (b). Panels d, e, and f show selected curves from a, b, and c, respectively, fitted to eq 18 (dashed lines). The sample masses used in the particular experiments shown were $13.3 \mathrm{mg}$ (a), $12.3 \mathrm{mg}$ (b), and 9.1 mg (c). Data points were recorded each second and are shown as solid lines. To discriminate between the lines every 50th data point is shown as a symbol.

diffusion coefficient (Figure 4a) indicated that a portion of ions had entered further into the polymer than when lower oxidation potentials were used. Since the number of ions incorporated in the sample is rather independent of oxidation potential, Figure 5 , this evidently means that the incorporated glutamate ions are more separated from each other when higher oxidation potentials were applied to the PPy/PMo composite. A lower anion density most likely results in less swelling (Figure 1a) of the polymer network during anion incorporation and thus less contraction during anion extraction. This could explain the higher portion of immediately mobile anions in the glutamate containing PPy/ PMo composite after oxidation at high potentials.

From Figure 4c it is evident that the frequency by which the initially trapped anions are released decreases with increasing oxidation potential applied prior to reduction, excepting the release frequency describing the reduction after glutamate incorporation in the $\mathrm{PPy} / \mathrm{FeCl}_{3}$ composite at $+0.7 \mathrm{~V}$, which was previously found to be a too low oxidation potential to fully oxidize the sample. Hence, a higher oxidation potential applied prior to reduction results in a stronger entrapment of the portion 


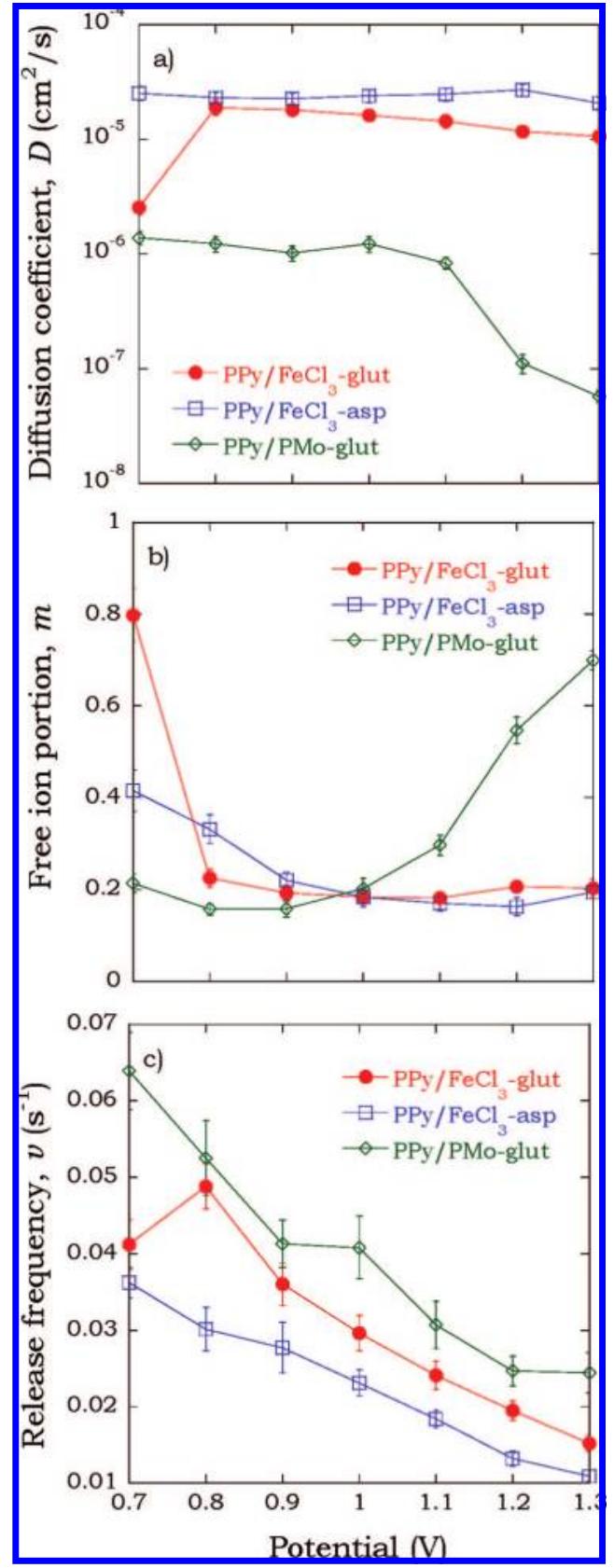

Figure 4. Diffusion coefficient (a), free portion of ions (b), and release frequency (c) vs anodic potential applied prior to reduction as obtained from curve fits similar to those in parts $\mathrm{d}-\mathrm{f}$ of Figure 3 for the three sample anion combinations under study. Error bars denote absolute deviations over three curve fits.

of anions that are initially trapped in the contracting polymer network. It is further evident from Figure $4 \mathrm{c}$ that the highest trap release frequencies are obtained for the anion release from the glutamate containing PPy/PMo composite. This can most likely be explained by the nanostructure of this composite, which is expected to contain holes that are about $10 \AA$ in size and thus considerably larger than those in the $\mathrm{PPy} / \mathrm{FeCl}_{3}$ composite. ${ }^{25}$ One further observes from Figure $4 \mathrm{c}$ that the trap release frequency is lower for the reduction of the aspartate containing $\mathrm{PPy} / \mathrm{FeCl}_{3}$ composite as compared to the glutamate containing counterpart. Since the glutamate anion has a slightly larger molecular mass than the aspartate anion this finding cannot be explained by a size effect as one would expect the smaller anion to be more easily released. The experiments with the aspartate and the glutamate anions were carried out at a $\mathrm{pH}$ of 10.4 and

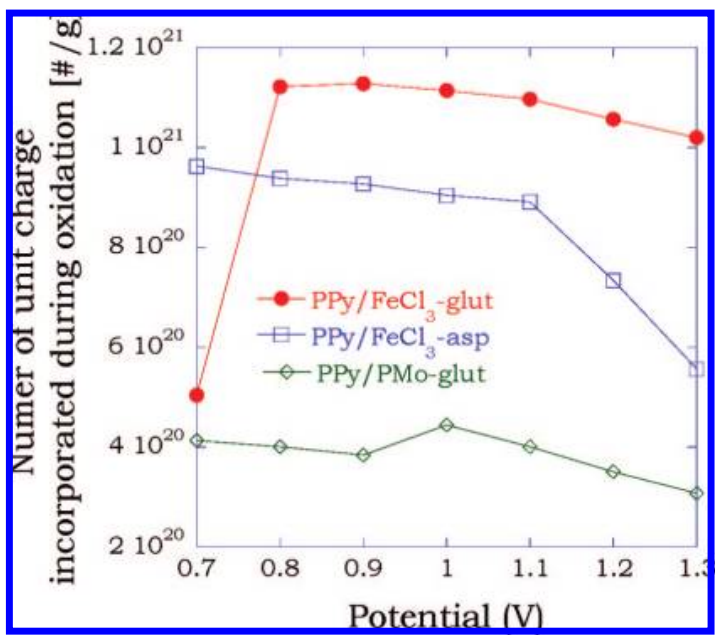

Figure 5. Number of ions incorporated in the composites under study during the anodic polarization preceding the cathodic process displayed in Figure 3.

9.0, respectively. By consideration that both aspartic and glutamic acids contain two $\mathrm{COOH}$ groups, at $\mathrm{pH} 10.4$ the aspartate solution should contain a mixture of doubly charged and singly charged anions since the $\mathrm{p} K_{\mathrm{a}}$ for the aspartate $\mathrm{R}-\mathrm{NH}_{3}{ }^{+}$ group is $10.0 .{ }^{37}$ The dominating species in the case of glutamate should, on the other hand, be the singly charged anion as the corresponding $\mathrm{p} K_{\mathrm{a}}$ for the $\mathrm{R}-\mathrm{NH}_{3}{ }^{+}$group is $9.9 .{ }^{37}$

The aspartate anion, on the average, carrying a larger negative net charge than the glutamate anion most likely causes the trapped aspartate ions to become more strongly bound to the positively charged polymer during oxidation (cf. Figure 1a) which may explain the lower release frequency for this type of ion.

It is obvious from the above presented results that the polymers exhibit a clear memory effect as the response to a cathodic potential of constant magnitude produces different responses depending on the magnitude of the preceding anodic potential. As seen from Figure 5, the magnitude of the applied anodic potential determines the extent of oxidation and thus the quantity of anions absorbed. During oxidation the positively charged polymer chains repel each other and open channels and cavities into which anions can be absorbed resulting into polymer network swelling. During reduction, however, when the charge is removed, the polymeric chains diffuse into the free volume left by the anions, promoting the closure of the polymeric entanglement during the time span of cathodic polarization. From the results presented herein, it can be concluded that anion diffusion out from the polymer network is being hindered at the initial stages, probably due to the network contraction, and that the experimentally observed temporary increase in the absolute value of the current can be analytically described by a trap release process similar to that observed in other physical systems involving diffusion of ions in solids.

\section{Summary and Conclusions}

In the present work we analyzed the movement of anions during cathodic polarization as a function of the preceding anodic polarization of varying magnitude in novel composite materials. The composites were found to exhibit memory effect as the response to a cathodic polarization of constant magnitude produced different responses depending on the magnitude of the preceding anodic potential. After the application of a 
cathodic potential, the reduction current curves were found to initially increase in magnitude followed by a monotonic decay. This response is similar to that observed when ions move from traps at the interface between solid electrode and dielectric materials. By fitting the recorded reduction current curves to a theoretical model derived herein, detailed information giving fundamental insight about the ionic movement in the conducting polymer layer of the composites under study could be obtained accounting both for the fraction of temporarily trapped ions and those initially mobile. These results may in turn be used to further optimize the materials properties of conducting polymer systems aimed for specific electrochemical ion exchange processes.

Acknowledgment. The equipment used in the present study was financially supported by a grant from the Knut and Alice Wallenberg Foundation.

\section{References and Notes}

(1) MacDiarmid, A. G. Angew. Chem., Int. Ed. 2001, 2581.

(2) Li, X. G.; Huang, M. R.; Duan, W.; Yang, Y. L. Chem. Rev. 2002, $102,2925$.

(3) Wang, L. X.; Li, X. G.; Yang, Y. L. React. Funct. Polvm. 2001, 47,125 .

(4) Saidman, S. B. Electrochim. Acta 2003, 48, 1719

(5) Silk, T.; Tamm, J. Electrochim. Acta 1996, 41, 1883.

(6) Yang, H.; Kwak, J. J. Phvs. Chem. B 1997, 101, 774.

(7) Yongfang, L.; Renyuan, Q. J. Electoanal. Chem 1993, 362, 267.

(8) Tamm, J.; Johanson, U.; Marandi, M.; Tamm, T.; Tamm, L. Russ. J. Electrochem. 2004, 40, 344.

(9) Foulds, N. C.; Lowe, C. R. Anal. Chem. 1988, 60, 2473.

(10) Rajesh; Pandey, S. S.; Takashima, W.; Kaneto, K. Curr. Appl. Phvs. 2005, 5, 184

(11) Xiao, Y.; Li, C. M.; Liu, Y. Biosens. Bioelectron. 2007, 22, 3161.

(12) Fabre, B.; Simonet, J. Coord. Chem. Rev. 1998, 178-180, 1211

(13) Collins, G. E.; Buckley, L. J. Synth. Met. 1996, 78, 93.

(14) Kaner, R. B., Knobler, C. M., Guo, H. Chiral recognition polymer and its use to separate enantiomers; United States Patent 6,265,615, USA, 2001.
(15) Pich, A.; Lu, Y.; Adler, H. J. P. Polvmer 2006, 47, 6536.

(16) van de Leur, R. H. M.; van der Waal, A. Synth. Met. 1999, 102, 1330.

(17) Aoki, K.; Kawase, M. J. Electoanal. Chem 1994, 377, 125.

(18) Tezuka, Y.; Aoki, K.; Yajima, H.; Ishii, T. J. Electoanal. Chem 1997, 425, 167.

(19) Tezuka, Y.; Ohyama, S.; Ishii, T.; Aoki, K. Bull. Chem. Soc. Jpn. 1991, 64, 2045.

(20) Vorotyntsev, M. A.; Aoki, K.; Heinze, J. Russ. J. Electrochem. 2003, 39, 182 .

(21) Marque, P.; Roncali, J. J. Phvs. Chem. 1990, 94, 8614.

(22) Smela, E.; Inganäs, O.; Pei, Q. B.; Lundström, I. Adv. Mater. 1993, 5,630 .

(23) Otero, T. F.; Grande, H.; Rodriguez, J. Electrochim. Acta 1996, 41,1863 .

(24) Mihranyan, A.; Nyholm, L.; Garcia-Bennett, A. E.; Strømme, M. J. Phys. Chem. B 2008, 112, 12249 .

(25) Razaq, A.; Mihranyan, A.; Welch, K.; Nyholm, L.; Strømme, M. J. Phvs. Chem. B 2009, 113, 426.

(26) Gelin, K.; Mihranyan, A.; Razaq, A.; Nyholm, L.; Strømme, M. Electrochim. Acta 2009, 54, 3394.

(27) Mihranyan, A.; Edsman, K.; Strømme, M. Food Hvdrocolloids 2007, 21, 267.

(28) Churchill, R. V. Operational mathematics, 3rd ed.; McGraw-Hill: New York, 1971.

(29) Mihranyan, A.; Llagostera, A. P.; Karmhag, R.; Strømme, M.; Ek, R. Int. J. Pharm. 2004, 269, 433.

(30) Otero, T. F.; Grande, H.; Rodriguez, J. Synth. Met. 1996, 76, 285.

(31) Vuki, M.; Kalaji, M.; Nyholm, L.; Peter, L. M. J. Electoanal. Chem

1992, 332, 315 .

(32) Strømme Mattsson, M.; Isidorsson, J.; Lindström, T. J. Electrochem. Soc. 1999, 146, 2613.

(33) Greeuw, G.; Hoenders, B. J. J. Appl. Phvs. 1984, 55, 3371.

(34) Liljegren, G.; Pettersson, J.; Markides, K. E.; Nyholm, L. Analvst 2002, 127, 591.

(35) Marcus, Y. Ion Solvation; Wiley: New York, 1985.

(36) Chu, L. Z.; Yuanming; Xuan, M.; Tang, Y. Chem. J. 2002, 4 (6), 26.

(37) Aylward, G.; Finlay, T. SI Chemical Data, 5th ed.; Wiley: New York, 2002.

JP9002627 